\title{
Peripheral blood mononuclear cell gene array profiles in female patients with involuntary bladder contractions
}

This article was published in the following Dove Press journal:

Advances in Genomics and Genetics

2 June 2011

Number of times this article has been viewed

\section{Wellman Cheung' \\ Mark J Bluth' \\ Sohail Khan² \\ Christopher Johns ${ }^{2}$ \\ Martin H Bluth ${ }^{3}$}

'State University of New York Downstate Medical Center, Brooklyn, NY, USA; ${ }^{2}$ Cold Spring Harbor Laboratory - Microarray Shared Resource, Cold Spring Harbor, NY, USA; ${ }^{3}$ Wayne State University School of Medicine, Detroit, MI, USA
Correspondence: Martin H Bluth Wayne State University School of Medicine, Department of Pathology, 8203 Scott Hall, 540 E Canfield, Detroit, MI 4820I, USA

$\mathrm{Tel}+|3| 37451869$

$\mathrm{Fax}+\mathrm{I} 3139934325$

Email mbluth@med.wayne.edu
Background: Patients with urgency represent a group of incontinence sufferers whose diagnosis remains difficult to establish. Urodynamic testing demonstrating involuntary bladder contraction provides objective confirmation but represents an invasive approach. We have previously demonstrated that peripheral blood mononuclear cells (PBMC) can provide a reporter function in solid organ disease toward biomarker discovery. Here we investigated the utility of using PBMC as marker for patients with confirmed involuntary bladder contraction.

Methods: Fifteen female patients were evaluated for involuntary bladder contractions and stress urinary incontinence as demonstrated by urodynamics and also assessed for pelvic prolapse, stress incontinence by history, bladder neck dysfunction, and bladder capacity. PBMC were obtained from patients' whole blood, and RNA was subjected to microarray gene chip analysis.

Results: Microarray analysis revealed that eleven genes were differentially regulated (five upregulated and six downregulated). Of these, PGRMC1 (progesterone receptor membrane component 1), EIF2S3 (eukaryotic initiation factor), C3AR1 (complement receptor), and three unknown genes were downregulated. Upregulated genes included MYOM2 (myomesin M-protein), a cytoskeletal protein; KTN1 (kinectin); and AAK 1 (AP2 associated kinase).

Conclusions: Microarray analysis revealed many genes that were differentially regulated in PBMC from patients with involuntary detrusor contractions. These genes may be important in regulating structural integrity of bladder and supporting tissues. These data suggest that PBMC can provide a reporter function for patients with involuntary bladder contractions and may serve toward biomarker discovery for novel diagnostic markers and/or the ability to monitor response to therapy.

Keywords: microarray, overactive bladder, incontinence, biomarker

\section{Introduction}

Urologic disease within the category of incontinence, including overactive bladder $(\mathrm{OAB})$ and urgency, represents a diverse group of conditions that are often difficult to treat as well as diagnose. Patient histories are often employed as a screening modality ${ }^{1,2}$ and urodynamic testing demonstrating involuntary bladder contraction provides objective confirmation but represents an invasive approach. Furthermore, treatment can be implemented in a trial and error fashion until patient relief is obtained. In an effort to elucidate biomarkers for incontinence-related diseases toward better understanding the pathophysiology of disease, we have previously demonstrated that peripheral blood mononuclear cells (PBMC) can provide a reporter function in solid organ diseases including pancreatitis and overactive bladder. ${ }^{3,4}$ In those studies, we employed the ability of the immune system to "cross talk" with the affected organ to 
provide a mirrored reflection of solid organ disease toward biomarker discovery. Since female health issues, such as incontinence and overactive bladder, represent an increasing concern with respect to quality-of-life, psychosocial, and economic dynamics, ${ }^{5-8}$ and treatment modalities and many diagnoses are often based on patient reporting, biomarkers for such disease states are needed for objective diagnosis of urological diseases. Here, we investigated the utility of using PBMC as a biomarker discovery platform for female patients with confirmed involuntary bladder contraction.

\section{Methods}

\section{Patient specimens}

Peripheral blood (4-5 mL total) was obtained from 15 female patients - average age 51.6 (range 35-63) - with involuntary bladder contractions treated at the Urology Clinic at State University of New York Downstate Medical Center. This study was approved by the Institutional Review Board. All patients completed a validated questionnaire (OAB V8) $)^{1,2,9}$ for screening of incontinence, and had hallmarks of such including frequency (voiding q2h or more), nocturia 2 or more times at night, and had post void urgency. Further, all patients in this study showed detrusor overactivity during urodynamics and a maximum functional capacity of $<250 \mathrm{~mL}$. All patients had severe frequency, nocturia, and urgency and were objectively assessed for incontinence by video urodynamics. All patients were diagnosed as having urinary incontinence. Patients were also evaluated for involuntary bladder contractions and stress urinary incontinence as demonstrated by urodynamics, pelvic prolapse, stress incontinence by history, bladder neck dysfunction, and bladder capacity as previously reported; ${ }^{4}$ bladder neck dysfunction was defined as delayed opening of bladder neck of more than 10 seconds on urodynamics. Blood obtained from three female volunteers (average age 52), served as healthy controls. Patients and controls did not have any history of recent infection or inflammatory disease and were not using immunosuppressing or immunomodulating therapy. Patients with a history of recent surgery, infection, and positive valsalva leak point pressure or negative for involuntary bladder contraction as demonstrated by urodynamics were excluded. All samples were collected before any invasive procedures (urodynamics, surgery). Patient consent for specimens and internal review board approval was obtained.

\section{Blood collection and processing}

Blood was collected into EDTA Monoject tubes (Sherwood Medical, St Louis, MO) and retained for up to 2 hours at room temperature. Whole blood was diluted (1:1) with
RPMI-1640 (GIBCO BRL Life Technologies, Grand Island, NY) and PBMC were separated from whole blood on FicollPaque (Pharmacia, Uppsala, Sweden) gradients (density, 1.077) in a 1:1 ratio. PBMC were washed twice in Dulbecco's phosphate buffered saline (Sigma-Aldrich, St Louis, MO) (pH 7.4, GIBCO-BRL). Viability was $\geq 98 \%$, as judged by trypan blue dye exclusion. ${ }^{4}$

\section{Nucleic acid purification and microarray analysis}

Total RNA was extracted from PBMC, as previously described, ${ }^{3,4}$ using the RNeasy mini kit and eluted using an RNeasy spin column (Qiagen, Inc, Valencia, CA) according to manufacturer's recommendations. RNA quality was assessed on an Agilent 2100 Bioanalyzer, RNA nano 6000 Series II Chips (Agilent, Palo Alto, CA). Samples were assessed by a RIN score; samples with a score of $>7.5$ were passed and quantity was assessed by Nanodrop ND-1000 (Nanodrop Technologies, Wilmington, DE). Total RNA was amplified by a modified Eberwine technique, using a Message amperes II kit (ABI, Austin, TX). The amplified product was assessed for a normal size distribution using the Agilent 2100 Bioanalyzer RNA 6000 nano Series II Chips. Samples were then prepared for hybridization, hybridized, washed, and scanned according to the manufacturer's instructions on U133A 2.0 GeneChip (Affymetrix, Santa Clara, CA). Affymetrix QC metrics were used to pass the image data. The U133A 2.0 gene chip was based on sequences submitted to the UniGene database (Build 133, April 20, 2001) profiling over 18,400 transcripts representing 14,500 well-characterized genes.

\section{Statistical analysis}

Differential expression analysis was performed using the limma software package from Bioconductor (Seattle, WA).,10 A linear model is fitted to model the systematic part of the data. For statistical analysis, limma uses empiric Bayes method to moderate the standard errors of the estimated logfold change. Patient and differential expression was defined as a change of at least two-fold vs respective controls. The significance level was set at greater than two-fold change among groups $(P \leq 0.05)$.

\section{Results}

All 15 patients demonstrated involuntary bladder contractions by urodynamics testing. Of the 15 patients assessed, 8 were positive for involuntary bladder contractions, however, they were negative for valsalva leak point pressure on urodynamics. Other patient characteristics included patients' average age of 
51.6 (range 35-66), where most had pelvic prolapse (75\%) and stress incontinence by history (63\%). Thirty-eight percent of patients had bladder neck dysfunction and all patients had normal to low bladder capacity. Microarray analysis was performed and all genes that were differentially regulated a minimum of two-fold difference from controls were captured (Figure 1). Microarray analysis revealed that eleven genes were differentially regulated (five upregulated and six downregulated) (Figure 2). Of these, PGRMC1 (progesterone receptor membrane component 1), EIF2S3 (eukaryotic initiation factor), C3AR1 (complement receptor), and three unknown genes were downregulated. Upregulated genes included MYOM2 (myomesin M-protein), a cytoskeletal protein involved in structural integrity.

A

A $\quad$ M versus $P$ values all females

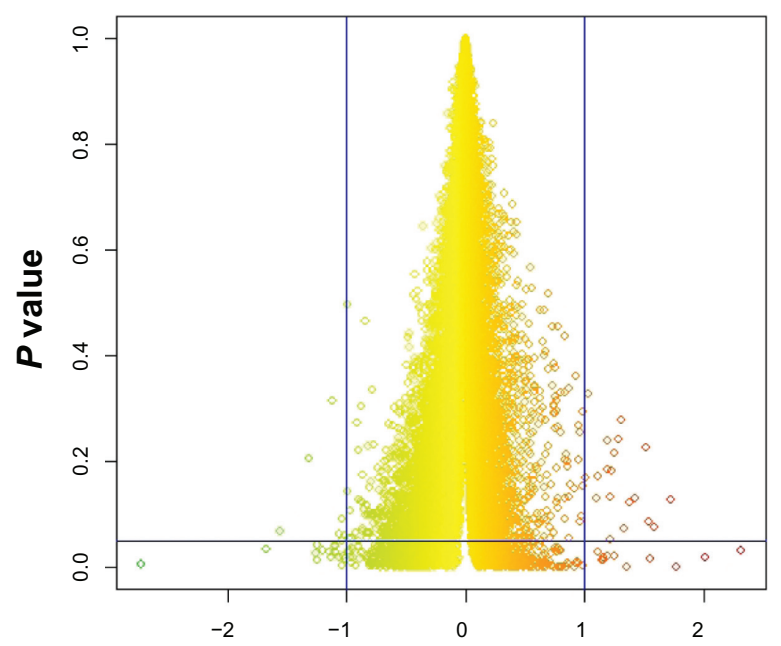

B

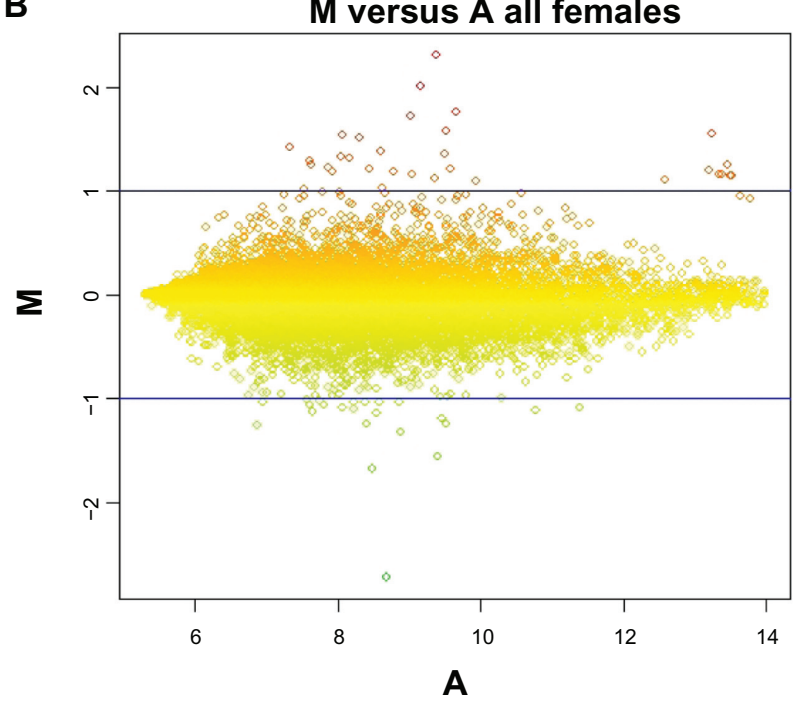

Figure I $M$ versus $P$ (panel $\mathbf{A}$ ) and $M$ versus $A$ plots (panel $\mathbf{B}$ ) for female data sets. These data represent the $\log 2$ ratio versus the average ratio. These plots give a quick overview of the distribution of the data. Genes that demonstrate $\geq 2$ fold (above $\log 2=$ I or below -1 ) were selected.

\section{Discussion}

Women's health-related diseases, including overactive bladder and incontinence, remain a challenge with respect to diagnosis and treatment. Further, the psychosocial aspects of such diseases can often affect quality of life for female patients and promote reclusive behavior and/or depression for fear of embarrassment. ${ }^{2,5-8}$ The economic burden on the health-care system is also a concern in light of the aging population. ${ }^{6,7}$ Options for disease management can range from therapy to surgical intervention and often vary according to disease presentation and urological comorbidities. ${ }^{8}$ Further, medications are often administered empirically and are modified based on patients' subjective responses. Urodynamics testing represents an objective test for diagnosis of incontinence. However, this approach is invasive, costly, requires appropriate skill to perform, and is not available in all health-care clinics. To date there are no diagnostic blood tests for incontinence. As such, a blood-based approach would be very useful for disease diagnosis and response management.

We have previously demonstrated that PBMC can serve a "reporter" function for biomarkers of acute pancreatitis and, as such, could identify unique genes expressed during pancreatitis. ${ }^{3}$ In an effort to adapt our leukocyte-based biomarker discovery to human disease, we applied microarray methodology to patients suffering from overactive bladder ${ }^{4}$ in those studies PBMC obtained from overactive bladder patients were analyzed. Microarray analysis revealed that 16 genes were differentially regulated (eight upregulated and eight downregulated) in all patients with overactive bladder compared with healthy controls. Furthermore, gender-based analysis demonstrated 74 genes differentially regulated in males (25 upregulated and 49 downregulated), and 30 in females (13 upregulated and 17 downregulated). Of these, PDGF; MFAP3L, a microfibrillar-associated protein; and TPM1 (tropomyosin) were downregulated in all sets analyzed.

Although the use of microarray as a means of elucidating gene changes involved in the pathophysiology of incontinence-type disease have been described, ${ }^{11,12}$ such studies utilize tissues such as vaginal and bladder mucosae for gene array studies. Obtaining these tissues dictates that an invasive approach be taken and would therefore be limited. The ability to adapt this technology to peripheral blood-derived nucleated cells would be ideal in that it would provide a noninvasive, easily accessible approach, without the side effects associated with organ biopsy.

In the current studies, we applied microarray mining methodology to include only female patients with involuntary 


$\begin{array}{lllll}\text { Genbank } & \text { Symbol } & \text { Fold change } & \boldsymbol{P} \text { value } & \text { Gene description } \\ \text { NM_014020 } & \text { LR8 } & 10.34577398 & 0.004118 & \\ \text { NM_018847 } & \text { HCA112 } & 4.516606059 & 0.017138 & \\ \text { NM_001415 } & \text { EIF2S3 } & 2.177418025 & 0.003966 & \text { Eukaryotic translation initiation factor 2, subunit 3 gamma, 52kDa } \\ \text { U62027 } & \text { C3AR1 } & 2.165511122 & 0.030888 & \text { Complement component 3a receptor 1 } \\ \text { AL049435 } & & 2.043812516 & 0.02356 & \\ \text { NM_006667 } & \text { PGRMC1 } & 2.02729272 & 0.049468 & \text { Progesterone receptor membrane component 1 } \\ \text { AA868898 } & \text { ZNF266 } & 2.0286798 & 0.002062 & \text { Zinc finger protein 266 } \\ \text { NM_004986 } & \text { KTN1 } & 2.332635869 & 0.039354 & \text { Kinectin 1 (kinesin receptor) } \\ \text { NM_014911 } & \text { AAK1 } & 2.740117433 & 0.021149 & \text { AP2 associated kinase 1 } \\ \text { BF968134 } & \text { PSMD14 } & 3.199986868 & 0.018246 & \text { Proteasome (prosome, macropain) 26S subunit, non-ATPase, 14 } \\ \text { NM_003970 } & \text { MYOM2 } & 6.164382435 & 0.020526 & \text { Myomesin (M-protein) 2, 165kDa }\end{array}$

Figure 2 Representative gene expression changes in PBMC obtained from female patients with involuntary bladder contractions compared with healthy female controls. Downregulated genes are shown in green and upregulated genes are shown in red. Abbreviation: PBMC, peripheral blood mononuclear cells.

bladder contractions as a means to focus on women's health-associated disease states with respect to incontinence. As in our previous studies, ${ }^{4}$ patients who had evidence of recent infection or immunological modulation, either reported or based on medication history, were excluded to obviate gene changes resulting from inflammatory responses unrelated to their urological disease.

Microarray analysis revealed many genes that were differentially regulated in PBMC from patients with involuntary bladder contractions, including PGRMC1 (progesterone receptor membrane component 1), EIF2S3 (eukaryotic initiation factor), and C3AR1 (complement receptor), which were downregulated. These genes are of note since if their expression were also altered in the bladder and/or its supporting tissues this could result in changes in maintenance of the bladder's structural integrity.

By way of example, PGRMC1 is reported to be involved in sterol metabolism, or homeostasis and cell survival. ${ }^{13,14} \mathrm{~A}$ recent study by Wu et $a 1^{13}$ has also demonstrated the relationship of PGRMC1 on contraction/relaxation of human myometrium during pregnancy, which suggests its role in maintaining appropriate uterine tone where a decrease in PGRMC1 during term or preterm labor might shift the balance to a state of heightened uterine contractility. EIF23S, which has been shown to regulate the rate of protein translation and escapes $\mathrm{X}$-inactivation in both humans and mice, was found to have increased expression in the brain and liver of females when compared with males. ${ }^{15}$ Furthermore, C3AR1, a pleotropic molecule involved in the complement system, also reported as a causal gene for obesity and diabetes, ${ }^{16,17}$ was identified as a causal gene for aortic lesion size in females but not in males and, as such, has been proposed to be involved in altering atherosclerotic lesion susceptibility. ${ }^{18}$ Interestingly, C3AR1 was one of the differentially regulated genes identified in stroke patients that employed a similar PBMC-microarray mining approach. ${ }^{19}$

To this end, C3AR1 and PGRMC1 also represented genes that were similarly downregulated and PSMD14, KTN1, and AAK1 represented genes that were upregulated only in female patients with overactive bladder where their PBMC were subjected to the same leukocyte-based microarray mining algorithm, ${ }^{4}$ further suggesting an involvement of these genes in general female incontinence. Upregulated genes included MYOM2 (myomesin M-protein), which is a member of the M-protein group of cytoskeletal proteins involved in the cross-linking of myofilaments. Although M-proteins have been described in limb and eye muscles, ${ }^{20,21}$ and are probably involved in maintenance of muscular tone, the relationship of MYOM2 to bladder contractility and its role in incontinence remains to be defined.

Furthermore, this approach identified three as of yet unknown genes (NM_014020-LR8, NM_018487-HCA112, AL_049435). Although the function of these expression sequence tags (EST) are unknown, we have found similar elucidation of ESTs in our earlier studies of PBMC-derived biomarker discovery. ${ }^{3}$ Mapping the expression pattern of the genes identified through leukocyte-based microarray mining approaches in the clinical arena might help differentiate the patients who have varying disease severity. It is clear that confirmatory studies of these genes and their respective protein products are needed to determine the utility of using PBMC 
to elucidate genes involved in incontinence-related disease. However, this provides a unique approach toward screening and elucidating novel biomarkers in disease via interrogation of the immune system vs the conventional approach of obtaining tissue directly from the affected organ. Additional studies are needed that encompass patient variation and disease expression in conjunction with comparison of gene expression differences, where possible obtained from the affected solid organ, to determine the reality of genes involved in disease.

In conclusion, PBMC are an easily accessible source of blood leukocytes that can provide a reporter function for solid organ disease. Furthermore, this approach may identify as of yet unknown genes that may be involved in the pathogenesis of diseases. These data suggest that PBMC can provide a reporter function for patients with incontinence and may serve as a diagnostic marker and/or monitor response to therapy and, as such, contribute to biomarker discovery and disease management in women's health.

\section{Acknowledgment}

This study was supported by Astellas Pharma Global Development and GlaxoSmithKline.

\section{Disclosure}

The authors report no conflicts of interest in this work.

\section{References}

1. Cheung WW, Khan NH, Choi KK, Bluth MH, Vincent M. Prevalence, evaluation and management of overactive bladder in primary care. $B M C$ Fam Pract. 2009;10:8.

2. Cheung WW, Borawski D, Abulafia O, Vincent MT, Harel M, Bluth MH. Characterization of overactive bladder in women in a primary care setting. Open Access J Urol. 2011;3:1-6.

3. Bluth MH, Lin YY, Zhang H, Viterbo D, Zenilman ME. Gene expression profiles in cells of peripheral blood identify new molecular markers of acute pancreatitis. Arch Surg. 2008;143:227-233.

4. Cheung WW, Bluth MJ, Johns C, Kahn S, Lin YY, Bluth MH. Peripheral blood mononuclear cell gene array profiles in patients with overactive bladder. Urology. 2010;75:896-901.

5. Abrams P, Kelleher CJ, Kerr LA, Rogers RG. Overactive bladder significantly affects quality of life. Am J Manage Care. 2000;6:S580-S590.
6. Stewart WF, Van Rooyen JB, Cundiff GW, et al. Prevalence and burden of overactive bladder in the United States. World J Urol. 2003;20: 327-336.

7. Hu TW, Wagner TH, Bentkover JD. Estimated economic costs of overactive bladder in the United States. Urology. 2003;61:1123-1128.

8. Milsom I, Abrams P, Cardozo L, Roberts RG, Thüroff JW, Wein AJ. How wide-spread are the symptoms of an overactive bladder and how are they managed? A population-based prevalence study. BJU Int. 2001;87:760-766.

9. Coyne K, Margolis M, Zyczynski T, Elinoff V, Roberts RG. Validation of an OAB screener in a primary care patient population in the US. Poster presented at the ICS and IUCA 2004 Scientific Programme; 2004 August 25-27, Paris, France.

10. Smyth GK. Linear models and empirical Bayes methods for assessing differential expression in microarray experiments. Stat Appl Genet Mol Biol. 2004;3:Article 3.

11. Chen B, Wen Y, Zhang Z, Guo Y, Warrington JA, Polan ML. Microarray analysis of differentially expressed genes in vaginal tissues from women with stress urinary incontinence compared with asymptomatic women. Hum Reprod. 2006;21:22-29.

12. Chen B, Wen Y, Zhang Z, Wang H, Warrington JA, Polan ML. Menstrual phase-dependent gene expression differences in periurethral vaginal tissue from women with stress incontinence. Am J Obstet Gynecol. 2003;189:89-97.

13. Wu W, Shi SQ, Huang HJ, Balducci J, Garfield RE. Changes in PGRMC1, a potential progesterone receptor, in human myometrium during pregnancy and labour at term and preterm. Mol Hum Reprod. 2011 Apr;17(4):233-242. Epub 2010 Dec 2.

14. Lösel RM, Besong D, Peluso JJ, Wehling M. Progesterone receptor membrane component 1: many tasks for a versatile protein. Steroids. 2008;73:929-934.

15. Xu J, Watkins R, Arnold AP. Sexually dimorphic expression of the $\mathrm{X}$-linked gene Eif2s3x mRNA but not protein in mouse brain. Gene Expr Patterns. 2006;6:146-155.

16. Schadt EE, Lamb J, Yang X, et al. An integrative genomics approach to infer causal associations between gene expression and disease. Nat Genet. 2005;37:710-717.

17. Yang X, Deignan JL, Qi H, et al. Validation of candidate causal genes for obesity that affect shared metabolic pathways and networks. Nat Genet. 2009;41:415-423.

18. Yang X, Peterson L, Thieringer R, et al. Identification and validation of genes affecting aortic lesions in mice. J Clin Invest. 2010;120: 2414-2422.

19. Grond-Ginsbach C, Hummel M, Wiest T, et al. Gene expression in human peripheral blood mononuclear cells upon acute ischemic stroke. J Neurol. 2008;255:723-731.

20. Agarkova I, Schoenauer R, Ehler E, et al. The molecular composition of the sarcomeric M-band correlates with muscle fiber type. Eur J Cell Biol. 2004;83:193-204.

21. Porter JD, Merriam AP, Gong B, et al. Postnatal suppression of myomesin, muscle creatine kinase and the $\mathrm{M}$-line in rat extraocular muscle. J Exp Biol. 2003;206:3101-3112.

\section{Publish your work in this journal}

Advances in Genomics and Genetics is an international, peer reviewed, open access journal that focuses on new developments in characterizing the human and animal genome and specific gene expressions in health and disease. Particular emphasis will be given to those studies that elucidate genes, biomarkers and targets in the development of new or improved therapeutic

\section{Dovepress}

interventions. The journal is characterized by the rapid reporting of reviews, original research, methodologies, technologies and analytics in this subject area. The manuscript management system is completely online and includes a very quick and fair peer-review system. Visit http://www.dovepress.com/ testimonials.php to read real quotes from published authors. 